\title{
External ventricular drainage alone versus endoscopic surgery for severe intraventricular hemorrhage: a comparative retrospective analysis on outcome and shunt dependency
}

\author{
Luca Basaldella, M.D., ${ }^{1}$ Elisabetta Marton, M.D., ${ }^{1}$ Alessandro Fiorindi, M.D., Ph.D., ${ }^{1}$ \\ Bruno Scarpa, Ph.D., ${ }^{2}$ Hadi Badreddine, M.D., ${ }^{1}$ and Pierluigi Longatti, M.D. ${ }^{1}$ \\ ${ }^{1}$ Department of Neurosurgery, Treviso Regional Hospital, University of Padova, Treviso; and ${ }^{2}$ Department of \\ Statistical Sciences, University of Padova, Italy
}

\begin{abstract}
Object. Massive intraventricular hemorrhages (IVHs) require aggressive and rapid management to decrease intracranial hypertension, because the amount of intraventricular blood is a strong negative prognostic predictor on outcome. Neuroendoscopy may offer some advantages over more traditional surgical approaches on outcome and may decrease the number of shunt procedures that need to be performed.

Methods. The authors retrospectively reviewed the clinical and radiological data in 96 patients treated for massive IVH who were admitted between January 1996 and June 2008 to the neurosurgery unit after undergoing emergency CT scanning. Forty-eight patients (Group A) were treated with endoscopic aspiration surgery using a flexible endoscope with a "freehand" technique. A historical group of 48 patients (Group B) treated using external ventricular drain (EVD) placement alone was used as a comparison. The authors compared the radiological results with the clinical outcomes at 1 year according to the modified Rankin Scale and the need for internal CSF shunt treatment in the 2 groups.

Results. Endoscopic aspiration did not significantly affect the outcome at 1 year as determined using the modified Rankin Scale. Patients who underwent endoscopy had an EVD in place for 0.18 days fewer than patients treated with an EVD alone. Patients undergoing external ventricular drainage alone had a 5 times greater chance of requiring a shunting procedure than those treated using neuroendoscopy and external ventricular drainage. Neuroendoscopy plus external drainage reduces shunting rates by $34 \%$ when compared with external drainage alone.

Conclusions. The reduction in internal shunt surgery encourages the adoption of neuroendoscopic aspiration of severe IVH as a therapeutic tool to decrease shunt dependency.

(http://thejns.org/doi/abs/10.3171/2012.1.FOCUS11349)
\end{abstract}

\section{Key Words • intraventricular hemorrhage • neuroendoscopy • ventriculoperitoneal shunt - external ventricular drainage}

$\mathrm{D}$ URING the past decade, there has been a considerable and increasing interest in the medical and surgical management of massive IVHs. ${ }^{4,8,16,20,26,36,37,51}$ Intraventricular blood brings about multiple noxious effects ${ }^{10,13}$ on the brain causing impairment of CSF circulation, ${ }^{29}$ intracranial hypertension, ${ }^{19}$ and acute ${ }^{14}$ or delayed hydrocephalus. ${ }^{15}$

Mortality estimates for IVH range from $50 \%$ to $80 \%$. The most common cause of IVH is spontaneous $\mathrm{ICH}^{6,18}$

\footnotetext{
Abbreviations used in this paper: $\mathrm{AVM}=$ arteriovenous malformation; EVD = external ventricular drain; GCS = Glasgow Coma Scale; $\mathrm{ICH}=$ intracranial hemorrhage; $\mathrm{ICP}=$ intracranial pressure; $\mathrm{IVH}=$ intraventricular hemorrhage; LOS = length of stay; $\mathrm{mRS}=$ modified Rankin Scale; $\mathrm{SAH}=$ subarachnoid hemorrhage; $\mathrm{VP}=$ ventriculoperitoneal.
}

followed by $\mathrm{SAH}{ }^{40}$ The incidence of IVH in intracerebral hemorrhage is roughly twice that in $\mathrm{SAH} .{ }^{18}$ Approximately $10 \%$ of patients suffering aneurysmal SAH and $40 \%$ of those suffering primary ICH experience IVH. ${ }^{6,18,40}$ At present, medical management of ICH and IVH revolves around the control of ICP. Despite medical management, mortality remains high, with only $38 \%$ of patients surviving the 1st year. ${ }^{6}$ Even with the best medical management, mortality rates have been reported to be as high as 50\%. These studies suggest that measures to control ICP through control of factors such as hydrocephalus have little effect. ${ }^{33}$ Massive IVH requires aggressive and rapid management to decrease intracranial hypertension. The amount of intraventricular blood is a strong prognostic factor,, 34 and its fast removal should be considered a priority. The immediate control of ICP by external ventricular drainage is a res- 
cue surgical action, ${ }^{21}$ but tetraventricular blood inundation should be managed using bilateral ventricular catheters, which frequently become obstructed by blood clots and need to stay in place for a longer period for blood washout. Naff et al. ${ }^{31}$ clearly demonstrated that the percentage of clot resolution is $10.8 \%$ per day and is independent from the initial clot volume, patient age and sex, type of underlying hemorrhage, and use of external ventricular drainage. ${ }^{24}$

The fibrinolytic system of CSF is limited. ${ }^{24}$ Blood may remain for weeks after hemorrhage; acute clots obstruct ventricular CSF pathways, and clot degradation products obstruct extraventricular CSF pathways. When present, blood degradation products continue to contribute to pa-

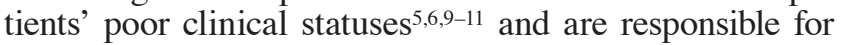
chronic shunt-dependent hydrocephalus in more than $30 \%$ of them. ${ }^{15-17,30}$ At present, reduction in the ventricular clot size seems to be the only method for reducing mortality rates after the ICH has stabilized. Many studies have demonstrated the independent effect of IVH on mortality. ${ }^{16-20}$ Neuroendoscopy may offer some advantages over more traditional surgical approaches such as EVD placement and intraventricular fibrinolysis and should be considered the gold-standard approach, especially when dealing with elderly patients in poor general health.

\section{Methods}

\section{Patient Population}

We retrospectively reviewed the demographic, clinical, and outcome data of a series of 96 patients treated for massive IVH between January 1996 and June 2008 (Table 1). All patients presented with spontaneous primary or secondary tetraventricular inundation, acute hydrocephalus, progressive worsening of neurological conditions, and/or coma. A CT scan was obtained within 3 hours of hospital admission, and the blood amount was quantified according

TABLE 1: Demographic and clinical characteristics

\begin{tabular}{lcc}
\hline \multicolumn{1}{c}{ Variable } & Group A & Group B \\
\hline sex & 28 & 23 \\
male & 20 & 25 \\
female & 1.4 & 0.92 \\
M/F ratio & & \\
age (yrs) & 57.8 & 56.4 \\
mean & $7-80$ & $14-78$ \\
range & & \\
etiology distribution (\%) & 39 & 36 \\
ICH & 29 & 50 \\
ruptured aneurysm & 17 & 10 \\
pure IVH & 8 & 4 \\
AVM & 6 & 0 \\
posterior fossa hemorrhage & 9.75 & 8.5 \\
mean admission Graeb score & 6.6 & 7.2 \\
mean admission GCS score & 12.1 & 13.9 \\
mean duration of EVD placement (days) & 13 & 19 \\
mean ICU LOS (days) &
\end{tabular}

to the Graeb score. ${ }^{14}$ Angiography was performed when a vascular malformation was suspected. Surgery was always performed in emergency conditions. Forty-eight patients underwent neuroendoscopic surgery (Group A), and the remaining 48 patients underwent EVD placement alone (control Group B). No fibrinolytic agents were used in either group. In patients with a vascular malformation as the likely cause of bleeding, the endoscopic procedure or the EVD placement was performed in close association with endovascular or surgical treatment. In all patients, clinical follow-up was assessed at 1 year according to the mRS score (see Fig. 3). ${ }^{\text {? }}$

Group A. Forty-eight patients (28 male and 20 female; male/female ratio 1.4) with a mean age of 57.8 years (range 7-80 years) were treated with endoscopic aspiration of intraventricular blood. All patients exhibited acute hydrocephalus on the initial CT scan as well as blood clots obstructing the third and fourth ventricles.

The IVH was a result of $\mathrm{ICH}$ in $39 \%$ of patients, a ruptured aneurysm in $29 \%$, primary IVH in $17 \%$, an AVM in $8 \%$, and a posterior fossa hematoma in $6 \%$ (Fig. 1). The severity of IVH was graded according to the Graeb scoring system: $65 \%$ of patients had a Graeb scale score greater than 10 and tetraventricular clots. The mean Graeb score was $9.75 \pm 2.7( \pm$ SD). The mean GCS score at the time of admission was 6.6. ${ }^{45}$ Sixty-three percent of patients were treated on the day of admission (within 24 hours from the onset of hemorrhage). The remaining $37 \%$ were treated in a delayed fashion (between 48 and 72 hours after onset). Especially in the initial period of training of the last author (P.L.), some of those patients who were admitted overnight were initially managed conservatively with an EVD if the surgeon was not available. Since that time, a second surgeon (A.F.) mastered the technique, and in the past 8 years we have been able to guarantee emergency treatment (within 24 hours) for all patients suitable for undergoing endoscopic aspiration of ventricular clots.

Group B. The records of 48 patients (23 male and 25 female; male/female ratio 0.92) with a mean age of 56.4 years (range 14-78 years) treated with external ventricular drainage for intraventricular blood removal were retrieved and matched from our institutional database as a historical

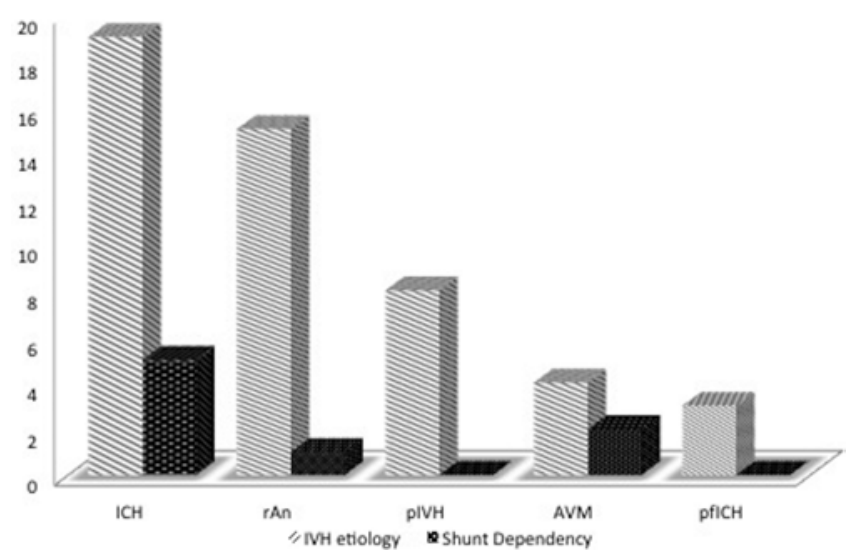

FIG. 1. Bar graph showing IVH etiology distribution and shunt dependency rates in Group A. pfICH = posterior fossa hematoma; pIVH = primary IVH; rAn = ruptured aneurysm. 
control group. All patients exhibited acute hydrocephalus on the initial CT scan.

The IVH was a result of $\mathrm{ICH}$ in $36 \%$ of patients, a ruptured aneurysm in $50 \%$, primary IVH in $10 \%$, and an AVM in $4 \%$ (Fig. 2). The severity of IVH was graded according to the Graeb scoring system. In $70 \%$ of patients the Graeb score was greater than 8 and tetraventricular clots were present; the mean Graeb score was $8.5 \pm 1.8$. The mean GCS score at admission was 7.2.

\section{Surgical Procedure}

Group A. Despite their clinical condition, all patients with IVH immediately underwent CT scanning, CT angiography, and digital subtraction angiography or MRI when deemed appropriate. Urgent blood work was obtained with special focus on prothrombin time and liver and kidney function. Preoperative management included identification and correction of coagulation disorders, administration of prophylactic anticonvulsants, controlling body temperature, and glycemia correction. The mean arterial pressure should be kept lower than $110 \mathrm{~mm} \mathrm{Hg}$ and systolic pressure lower than $150 \mathrm{~mm} \mathrm{Hg}$. For the endoscopic procedure, 2 types of flexible endoscopes were used with an external diameter of either $2.5 \mathrm{~mm}$ (Karl Storz) or $3.9 \mathrm{~mm}$ (Codman) and an operative length of $53 \mathrm{~cm}$. The internal diameter of the working channel was $1.2 \mathrm{~mm}$. For aspiration procedures, the entire working channel was used as a sucking device. The access was precoronal, $2 \mathrm{~cm}$ from lateral to the midline, through a $12-\mathrm{mm}$ bur hole (Figs. 4 and 5). The access side was chosen as the side of the lateral ventricle containing the largest amount of blood or the largest dilation. In cases with bilateral massive inundation, the access was bilateral. In the presence of a ventricular vascular malformation or aneurysm, an approach from that side was avoided. Lateral ventricle cannulation was achieved using a semirigid peel-away catheter, and systolic blood pressure was maintained at $120 \mathrm{~mm} \mathrm{Hg}$. An energetic intermittent manual aspiration was started using a rigid syringe connected to the operating channel of the endoscope. This action breaks down the clot and allows initial visualization in the ventricle. Once the choroidal plexus and Monro foramen were identified, the endoscope was advanced into the third ventricle, preferably into the right-sided bur hole,

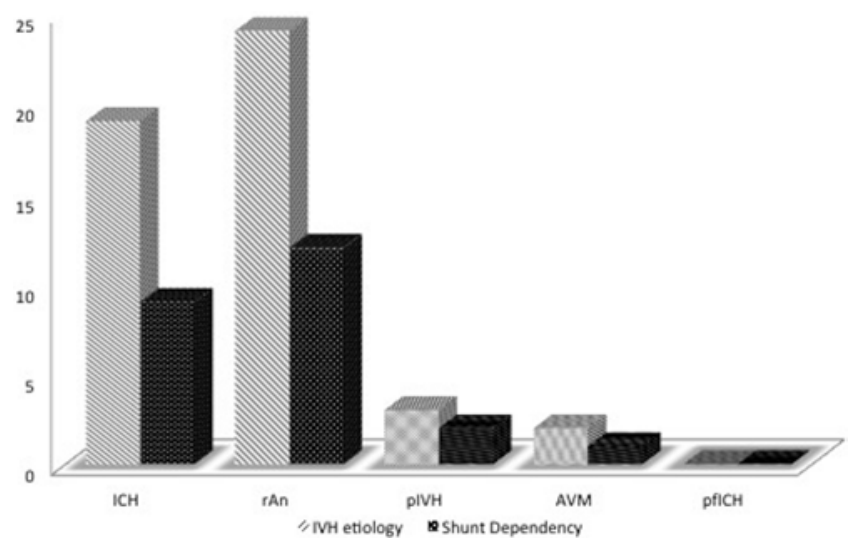

FIG. 2. Bar graph showing IVH etiology distribution and shunt dependency rates in Group B.

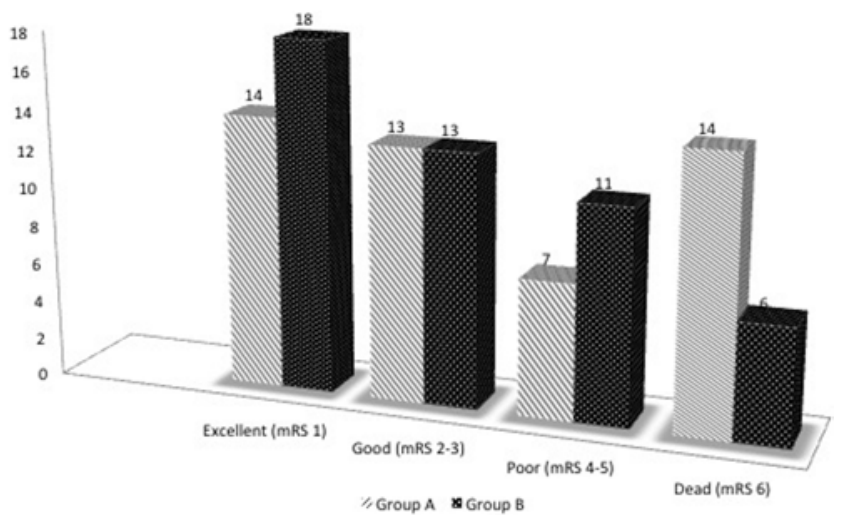

Fig. 3. Comparison of the 1-year mRS scores in Groups A and B.

and the procedure was repeated. Evacuation of the third ventricle paved the way to the aqueduct and to the fourth ventricle, where the aspiration-irrigation procedures were carefully performed, since the endoscope fills the entire diameter of the aqueduct and potentially dangerous local hypertension might easily be caused by excessive irrigation with Ringer solution. At the end of the procedure, an EVD was left in all patients for ICP monitoring and drainage (with a constant gradient of $15 \mathrm{~mm} \mathrm{Hg}$ ). The mean ICU LOS after the operation was 13 days. Endoscopy was successfully completed in all cases, and each patient received an EVD. There were no intraoperative deaths. The EVD was removed on the same day as the endoscopy procedure in 12 patients and after 1-25 days in the other patients (median 10 days).

Group B. An EVD was positioned in all patients on the 1st day. Bilateral EVDs were positioned in 35 of the 48 patients. The EVD was removed after 8-32 days (median 13.99 days), and all patients underwent catheter substitution 1 or more times during the ICU LOS. In this group, no thrombolysis with rtPA was performed to maintain homogeneity between the 2 groups. The mean ICU LOS after the operation was 19 days.

\section{Statistical Analysis}

Statistical models have been fitted to data to describe the specific effect of endoscopy once the effects of sex, age, and admission GCS score were eliminated. We fit a logistic model to measure the effects of available variables on the insertion of a VP shunt, where significant variables were selected by a backward stepwise procedure based on the Akaike information criterion.

Proportional odds models have been fitted to describe a connection between endoscopy and mRS score, which is a scoring variable that is ordinal in that its value increases when outcome is worse. Also in this case we selected significant variables by backward stepwise selection based on the Akaike information criterion. We also considered, as secondary outcomes of interest, the number of days that the EVD was required and the number of days spent in the neuro-ICU. Both these variables are quantitative, nonnegative, and right skew. We used the Box-Cox transformation to select the best function of these outcomes. In both cases the square root transform was chosen. Here too, in both cases, we implemented a backward stepwise procedure 
L. Basaldella et al.
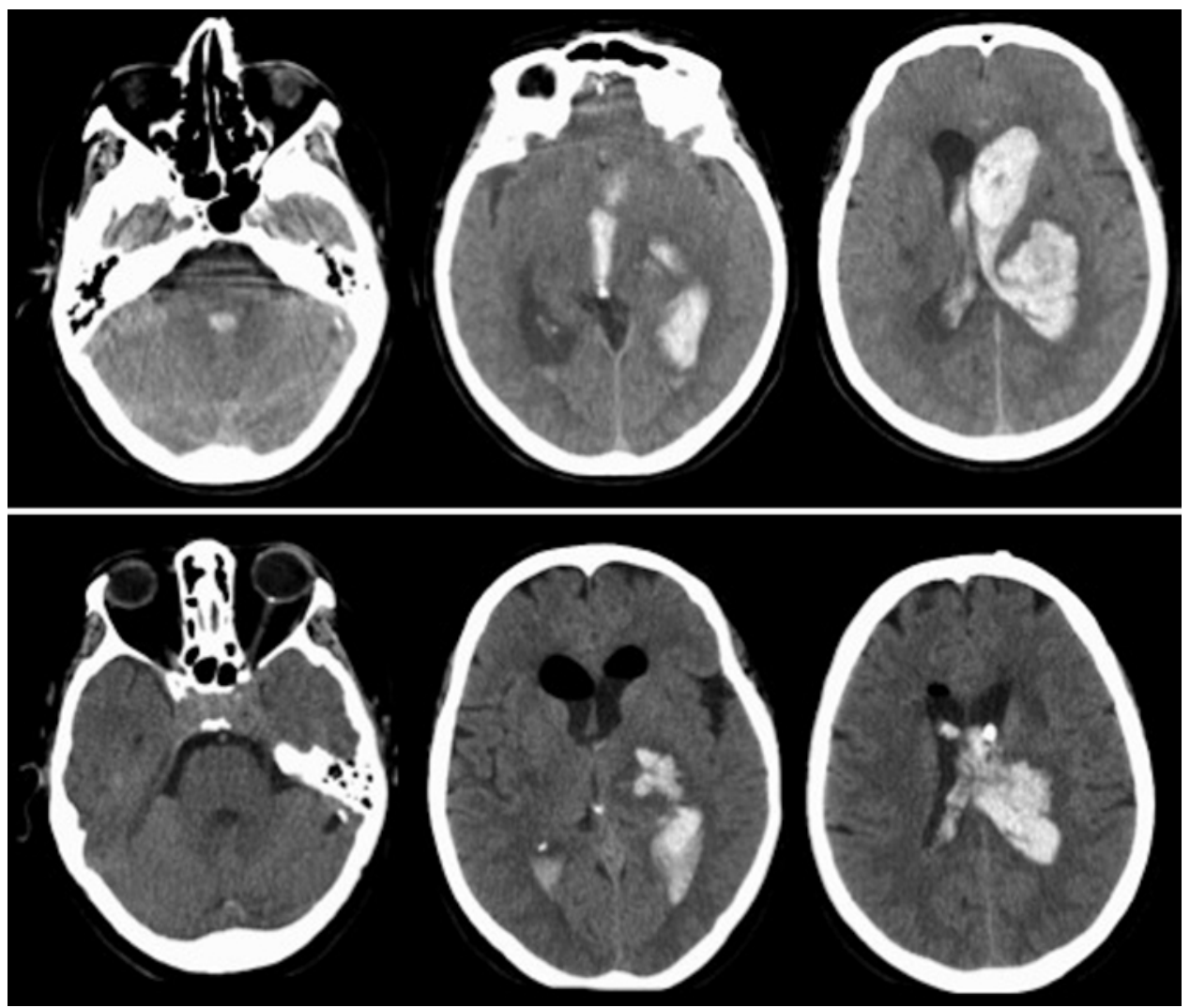

FIG. 4. Preoperative (upper) and postoperative (lower) CT scans of a spontaneous thalamic hemorrhage with tetraventricular clots.
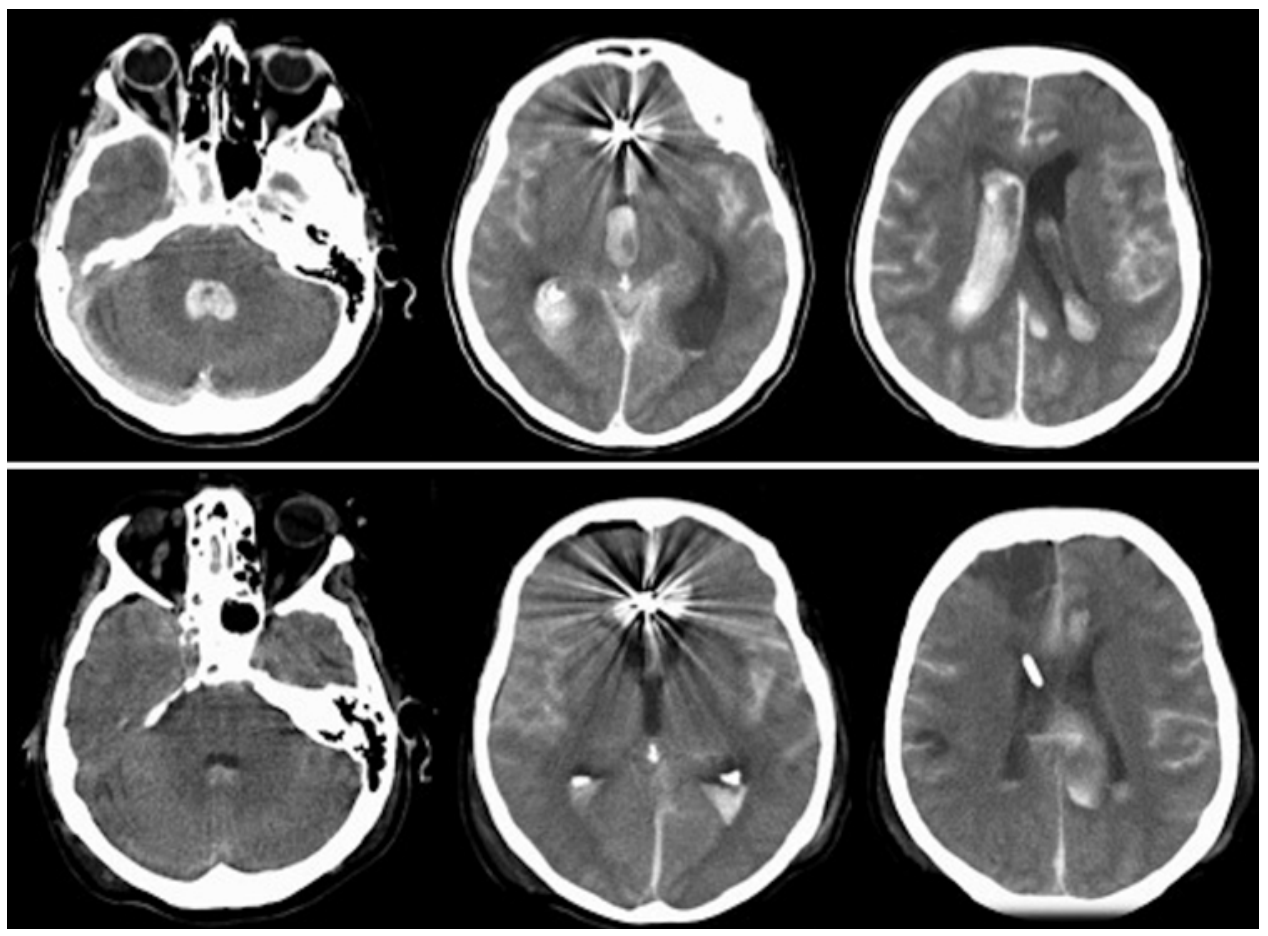

FIG. 5. Preoperative (upper) and postoperative (lower) CT scan of an anterior communicating artery aneurysm, treated with emergency coiling and subsequent endoscopic surgery plus external drainage. 
based on the Akaike information criterion to select the significant variables.

\section{Results}

A VP shunt was required by $17 \%$ of patients in Group A and by $50 \%$ of patients in Group B. The 1-year followup was recorded according to the mRS. Excellent outcome (mRS Score 0 or 1) was achieved in $29 \%$ of patients in Group A and in $37 \%$ of patients in Group B. A good outcome (mRS Score 2 or 3 ) was seen in $27 \%$ of patients in both groups. A poor outcome (mRS Score 4 or 5) was recorded in $14 \%$ and $23 \%$ of patients, and death (mRS Score 6) occurred in $29 \%$ and in $13 \%$ in Groups A and B, respectively (Table 2). The mRS results in an independent variable when compared with each group (Group A vs Group $\mathrm{B}, \mathrm{p}=0.2$; chi-square test).

Backward selection of the logistic model to estimate the effect of endoscopy on the need for a VP shunt revealed that age and admission GCS score were not significant when sex and endoscopy are included in the model. As shown in Table 3, patients who did not undergo endoscopy had an almost 5 times greater probability of requiring a shunt procedure than those who did.

The model describing the effects of endoscopy on mRS score provides different results. Here, only age and admission GCS score result were significant in describing the outcome; the fact that endoscopy was performed did not add any relevant information in predicting the mRS score. The same result of no effect of endoscopy on mRS score was obtained if this variable is recoded in the 4 classes scoring as in Table 2. We also did not find any effect of endoscopy when we relaxed the proportional odds hypothesis by fitting a multinomial model, by not considering the ordinal characteristic of the mRS score. We therefore conclude that there is not a specific effect of endoscopy once age and admission GCS score are considered in predicting the mRS score.

A significant effect of endoscopy is, instead, observed in both models predicting the number of days of external ventricular drainage and neuro-ICU LOS. The presence of endoscopy is significant $(\mathrm{p}=0.038)$ regardless of whether the other insignificant variables (sex, age, and admission GCS score) are included. Patients who underwent endoscopy spent about 0.18 days fewer in the ICU than those who underwent EVD placement alone (Table 4).

Similar results were obtained using the model when predicting the LOS in the neuro-ICU. In this case, only age and sex were not significant, whereas admission GCS score had a significant negative effect on this parameter.

TABLE 2: Modified Rankin Scale score distribution

\begin{tabular}{ccc}
\hline & \multicolumn{2}{c}{ No. of Patients (\%) } \\
\cline { 2 - 3 } mRS Outcome & Group A & Group B \\
\hline excellent (score 1) & $14(29)$ & $18(37)$ \\
good (score 2 or 3) & $13(27)$ & $13(27)$ \\
poor (score 4 or 5) & $7(14)$ & $11(23)$ \\
dead (score 6) & $14(29)$ & $6(13)$ \\
\hline
\end{tabular}

Patients who underwent endoscopy had about half a day (0.504) longer stay in the neurological ICU than the others (Table 4).

\section{Discussion}

The prognosis of IVH is affected by several factors, including age, sex, GCS score, presence of acute hydrocephalus, coagulopathy, origin of the bleeding, and tetraventricular inundation. These factors all play a critical role, but in the first hours after IVH, the sudden increase in ICP associated with initial bleeding may cause a significant reduction in cerebral blood flow, potentially leading to ischemia. ${ }^{28}$ The obstruction of CSF pathways and the mass effect associated with clots in the satellite hematoma and within the ventricles cause further damage. The fibrinolytic system of the CSF is limited, ${ }^{32}$ and blood may remain for weeks after hemorrhage. ${ }^{6,15,18}$ Delay of clot resolution leads to obstruction of extraventricular CSF pathways, contributing to the patient's poor clinical status. In the late stage, biochemical changes and blood degradation products are responsible for secondary damage to neurons and white matter. ${ }^{41,49}$ Hematoma and perihematoma regions after intracerebral hemorrhage are biochemically active environments known to undergo potent oxidizing reactions., ${ }^{911}$ After $\mathrm{ICH}$, amounts of unconjugated bilirubin in the hematoma can be substantial, as can levels of iron and hemoglobin. ${ }^{11}$ Oxidation of unconjugated bilirubin to yield bioactive molecules, such as BOXes (bilirubin oxidation products), is an important discovery, expanding the role of bilirubin in pathological processes seen after ICH. Blood degradation products are responsible for oxidative stress in brain surrounding the hematoma, and the spreading through the CSF may be responsible for late damage to the ventricle-facing structures. Thus, the primary goal of acute management for IVH should be the fast removal of intraventricular blood, rapid reversal of ventricular dilation, and normalization of ICP. All of these goals can be achieved immediately using the neuroendoscopic procedure, ${ }^{16,25,26,36,37,51}$ while complications often observed with other traditional approaches are minimized. ${ }^{33}$

\section{External Ventricular Drainage}

The initial management of IVH has included the placement of 1 EVD or more to allow the egress of blood and CSF from the ventricle system and decrease ICP. However, this approach alone is often not sufficiently effective in improving the poor prognosis of patients with severe IVH. ${ }^{4,15,21}$ Patency of the EVD is frequently difficult to maintain due to occlusion of the catheter by coagulated blood. Optimal treatment should also include removal and dissolution of the intraventricular hematoma itself. This would be expected to decrease ICP, increase cerebral perfusion, and minimize the chance of IVH-induced hydrocephalus resulting from obstruction of CSF drainage from the ventricular system. In selected cases, surgical evacuation of intraparenchymal and intraventricular hematomas has resulted in decreases in mortality rates and disability. ${ }^{12}$ However, surgery is invasive and is not always feasible. Survival after primary IVH is common, ${ }^{27}$ but it is accompanied by considerable morbidity. In general, patients with 


\section{Basaldella et al.}

TABLE 3: Estimates of the parameters for the final logistic model connecting endoscopy and VP shunt

\begin{tabular}{lccccc}
\hline \multicolumn{1}{c}{ Parameter } & Estimate & exp(estimate) & SE & z Value & $p$ Value \\
\hline base level (female of Group A) & -1.2284 & 0.293 & 0.4424 & -2.777 & 0.005 \\
additional effect for male & -0.7626 & 0.466 & 0.4725 & -1.614 & 0.106 \\
additional effect for Group B & 1.5771 & 4.841 & 0.4894 & 3.223 & 0.001 \\
\hline
\end{tabular}

secondary IVH fare worse than those with primary IVH. ${ }^{47}$ When an intracerebral hematoma extends into a portion of the ventricular system, mortality has been reported to be $32 \%-44 \% .14,22$ When IVH extends into all 4 ventricles, mortality has been reported as $60 \%-91 \% .^{4,6,12,18,40}$ With respect to the location of the hematoma causing the IVH, the worst prognosis is found with hematomas located in the thalamus. ${ }^{18}$ Predictors of poor outcome are GCS score on presentation and the volume of blood in the ventricle system. ${ }^{47,50}$ The degree of ventricular dilation, independent of the obstruction of CSF flow, has also been found to be a strong indicator for poor outcome. ${ }^{39}$

\section{Fibrinolytic Agents}

Investigators have sought to develop improved methods of treating patients with IVH, trying to expedite the resolution of the ventricular blood clots and improve morbidity and mortality in these patients by administering fibrinolytic agents directly into the ventricular system. The basic rationale for this treatment is to dissolve the blood clot within the ventricular system, since it continues to exert a deleterious effect on the brain. A major reason to consider the use of a fibrinolytic agent is to keep the EVD open so that blood and spinal fluid can continue to drain freely from the ventricular system. In 1990, Shen et al. ${ }^{43}$ reported on 4 patients with IVH who received [urokinase] $\mathrm{u}$-PA either as an intermittent infusion $(\mathrm{n}=2)$ or continuous infusion $(n=2)$, into the lateral ventricles through an EVD. All patients underwent bilateral EVD placement. Two patients had a good recovery, 1 had a severe disability, and 1 persisted in a vegetative state. All patients developed meningitis that was likely related to the continuous manipulation of the ventricular catheters. Rainov and Burkert ${ }^{39}$ reported results of $\mathrm{u}-\mathrm{PA}$ treatment that were found to be favorable. In their treatment and control groups, respectively, $15(94 \%)$ and $2(40 \%)$ patients had excellent or good outcomes (that is, no deficits or minor neurological deficits), and $1(6 \%)$ and $2(40 \%)$ patients had poor outcomes (that is, permanent vegetative state). No patient in the treatment group died, and 1 patient (20\%) in the control group died. Tush et al. ${ }^{48}$ have been the only authors to report complications encountered with intraventricular u-PA, including 1 case of rebleeding and 2 cases of ventriculitis (in a series of 5 patients). Coplin et al. ${ }^{11}$ conducted a retrospective cohort study in 1998, which included the largest number of patients thus far treated with intraventricular u-PA under a given protocol. Similar to other reports, the median time for clearance of blood from the third ventricle was 7.0 days and that from the lateral ventricles was 16.0 days. Thrombolytic therapy was found to result in significantly lower mortality $(31.8 \%$ vs $66.7 \%$; $p=0.03)$. Previous reports that have included a control group ${ }^{11}$ or comparison group ${ }^{39,43,48}$ in their analysis have similarly reported that treatment with intraventricular u-PA in patients with IVH reduces mortality by $30 \%-35 \%$. What is not clear from the literature, however, is whether such treatment results in an improved neurological outcome of the survivors. Shen et al., ${ }^{43}$ Akdemir et al., ${ }^{1}$ and Todo et al. ${ }^{46}$ reported good recovery with or without moderate disability in $50 \%-83 \%$ of the patients who received this treatment. However, 1 cohort study found that a larger proportion of the survivors remained in a vegetative state (31.8\% treatment group vs $11.1 \%$ control group). ${ }^{11}$ Andrews et al. ${ }^{2}$ concluded that clinical studies of fibrinolytic therapy for IVH have found a 30\%-35\% reduction in mortality with treatment, but, to date, they have not clearly documented improved neurological outcome of the survivors. Nieuwkamp et al. ${ }^{35}$ conducted a systematic review to compare conservative treatment, extraventricular drainage alone, and extraventricular drainage combined with fibrinolysis. They reported a poor outcome rate for conservative treatment of $90 \%$; that for extraventricular drainage was $89 \%$ (relative risk 0.98 [95\% CI 0.75-1.30]) and that for extraventricular drainage with fibrinolytic agents was 34\% (relative risk 0.38 [95\% CI 0.21-0.68]). In 2002 Lapointe and Haines ${ }^{23}$ concluded that there was suggestive and anecdotal evidence that the intraventricular administration of fibrinolytic agents in cases of IVH might be safe and of therapeutic value.

TABLE 4: Summary of the estimates for the final models relating endoscopy to square root of EVD placement and of neuro-ICU LOS

\begin{tabular}{lcccc}
\hline \multicolumn{1}{c}{ Model } & Estimate & SE & t Value & $p$ Value \\
\hline $\begin{array}{l}\text { square root of EVD } \\
\quad \text { Group A }\end{array}$ & 3.2564 & 0.1426 & 22.841 & $<0.0001$ \\
$\quad$ additional effect for Group B & 0.4244 & 0.2016 & 2.105 & 0.038 \\
$\begin{array}{l}\text { square root of neuro-ICU stay } \\
\text { base level (Group A) }\end{array}$ & 5.28544 & 0.50770 & 10.411 & $<0.0001$ \\
$\quad$ additional effect for each single GCS point at admission & 0.16497 & 0.07093 & 2.326 & 0.0222 \\
$\quad$ & 0.16497 & 0.27319 & 2.628 & 0.0100 \\
\hline
\end{tabular}




\section{Endoscopic Aspiration}

Neuroendoscopic approaches have been reported to produce positive outcomes in patients with IVH. ${ }^{8,16,25,36,37,51}$ In all cases, efficient removal of intraventricular clots was rapidly achieved. However, in 2 of these reports, use of a rigid endoscope required either a cumbersome biportal approach ${ }^{17}$ or an ultrasonic aspirator, ${ }^{38}$ with a high risk $(20 \%)$ of postoperative rebleeding. The use of a flexible instrument and the freehand technique, albeit offering a narrower operating channel, allows more complete cleansing of the third ventricle and navigation down to the fourth ventricle through the aqueduct. Persistence of blood in the fourth ventricle is related to poor outcomes in patients with IVH. ${ }^{42}$ Complete clearing of the aqueduct and the fourth ventricle is thus an important feature of this treatment because it rapidly establishes the physiological CSF dynamics.

Our experience with flexible endoscopic treatment of severe primary and secondary IVH did not demonstrate any infective complications or rebleeding, showing that this approach can be safe and effective and may favorably compare with more established approaches. ${ }^{34}$

\section{Conclusions}

Our experience with flexible endoscopic treatment of severe primary and secondary IVH shows that once certain measures are taken to minimize the risk of bleeding resulting from ruptured vascular malformations, this approach can be safe and effective and may compare favorably with more established approaches. Similar to other ventricular diseases, IVH can be treated successfully with flexible endoscopes, which may result in a very satisfactory, albeit challenging, approach for experienced neuroendoscopists. Flexible neuroendoscopy in our series was not associated with complications or rebleeding. Neuroendoscopy plus external ventricular drainage reduces shunting rates by $34 \%$ when compared with external ventricular drainage alone. Patients who undergo EVD placement have a 5 times higher probability of requiring an internal shunt than those treated with neuroendoscopy plus EVD placement. Neuroendoscopy plus EVD placement together do not significantly improve the 1-year mRS scores when compared with EVD placement alone. Neuroendoscopy plus EVD placement significantly reduces the need for postoperative external ventricular drainage days after surgery, potentially reducing infective complications. The limitation of this study lies in its retrospective nature. However, being aware of the selection biases in determining the treatment group due to differences in time epoch for the 2 treatments, we still think that the data from this study are lacking in the contemporary literature and may prompt a cooperative randomized trial to acquire Level A evidence data that may be adopted as guidelines in the management of an unsolved clinical problem. Reduction of the need for VP shunt procedures encourages the adoption of neuroendoscopic aspiration as a therapeutic tool in cases of massive IVH.

\section{Disclosure}

The authors report no conflict of interest concerning the materials or methods used in this study or the findings specified in this paper.
Author contributions to the study and manuscript preparation include the following. Conception and design: Basaldella, Longatti. Acquisition of data: Basaldella, Marton, Fiorindi, Badreddine. Analysis and interpretation of data: Basaldella. Drafting the article: Basaldella, Marton, Scarpa. Critically revising the article: Basaldella, Marton, Longatti. Reviewed submitted version of manuscript: Basaldella. Approved the final version of the manuscript on behalf of all authors: Basaldella. Statistical analysis: Scarpa. Study supervision: Basaldella, Fiorindi, Longatti.

\section{References}

1. Akdemir H, Selçuklu A, Paşaoğlu A, Oktem IS, Kavuncu I: Treatment of severe intraventricular hemorrhage by intraventricular infusion of urokinase. Neurosurg Rev 18:95-100, 1995

2. Andrews CO, Engelhard HH: Fibrinolytic therapy in intraventricular hemorrhage. Ann Pharmacother 35:1435-1448, 2001

3. Angelopoulos M, Gupta SR, Azat Kia B: Primary intraventricular hemorrhage in adults: clinical features, risk factors, and outcome. Surg Neurol 44:433-437, 1995

4. Aoki N: Treatment for intraventricular hemorrhage. J Neurosurg 75:494-495, 1991

5. Bakshi R, Kamran S, Kinkel PR, Bates VE, Mechtler LL, Belani SL, et al: MRI in cerebral intraventricular hemorrhage: analysis of 50 consecutive cases. Neuroradiology 41:401409, 1999

6. Bhattathiri PS, Gregson B, Prasad KS, Mendelow AD: Intraventricular hemorrhage and hydrocephalus after spontaneous intracerebral hemorrhage: results from the STICH trial. Acta Neurochir Suppl 96:65-68, 2006

7. Bonita R, Beaglehole R: Recovery of motor function after stroke. Stroke 19:1497-1500, 1988

8. Chen CC, Liu CL, Tung YN, Lee HC, Chuang HC, Lin SZ, et al: Endoscopic surgery for intraventricular hemorrhage (IVH) caused by thalamic hemorrhage: comparisons of endoscopic surgery and external ventricular drainage (EVD) surgery. World Neurosurg 75:264-268, 2011

9. Chen M, Regan RF: Time course of increased heme oxygenase activity and expression after experimental intracerebral hemorrhage: correlation with oxidative injury. J Neurochem 103:2015-2021, 2007

10. Clark JF, Loftspring M, Wurster WL, Beiler S, Beiler C, Wagner KR, et al: Bilirubin oxidation products, oxidative stress, and intracerebral hemorrhage. Acta Neurochir Suppl 105:7-12, 2008

11. Coplin WM, Vinas FC, Agris JM, Buciuc R, Michael DB, Diaz FG, et al: A cohort study of the safety and feasibility of intraventricular urokinase for nonaneurysmal spontaneous intraventricular hemorrhage. Stroke 29:1573-1579, 1998

12. de Weerd AW: The prognosis of intraventricular hemorrhage. J Neurol 222:46-51, 1979

13. Dull C, Torbey MT: Cerebral vasospasm associated with intraventricular hemorrhage. Neurocrit Care 3:150-152, 2005

14. Graeb DA, Robertson WD, Lapointe JS, Nugent RA, Harrison PB: Computed tomographic diagnosis of intraventricular hemorrhage. Etiology and prognosis. Radiology 143:91-96, 1982

15. Gross WP, Hesselmann V, Wedekind C: Development of chronic hydrocephalus and early cranial CT findings in spontaneous intracerebral/intraventricular hemorrhage. Zentralbl Neurochir 67:21-25, 2006

16. Hamada H, Hayashi N, Kurimoto M, Umemura K, Nagai S, Kurosaki K, et al: Neuroendoscopic removal of intraventricular hemorrhage combined with hydrocephalus. Minim Invasive Neurosurg 51:345-349, 2008

17. Horváth Z, Veto F, Balás I, Kövér F, Dóczi T: Biportal endoscopic removal of a primary intraventricular hematoma: case report. Minim Invasive Neurosurg 43:4-8, 2000

18. Huttner HB, Köhrmann M, Berger C, Georgiadis D, Schwab 


\section{Basaldella et al.}

S: Influence of intraventricular hemorrhage and occlusive hydrocephalus on the long-term outcome of treated patients with basal ganglia hemorrhage: a case-control study. J Neurosurg 105:412-417, 2006

19. Huttner HB, Staykov D, Bardutzky J, Nimsky C, Richter G, Doerfler A, et al: [Treatment of intraventricular hemorrhage and hydrocephalus.] Nervenarzt 79:1369-1370, 1372-1374, 1376, 2008 (Ger)

20. Huttner HB, Tognoni E, Bardutzky J, Hartmann M, Köhrmann M, Kanter IC, et al: Influence of intraventricular fibrinolytic therapy with rt-PA on the long-term outcome of treated patients with spontaneous basal ganglia hemorrhage: a case-control study. Eur J Neurol 15:342-349, 2008

21. Kiymaz N, Demir O, Cirak B: Is external ventricular drainage useful in primary intraventricular hemorrhages? Adv Ther 22:447-452, 2005

22. Lagares A, Putman CM, Ogilvy CS: Posterior fossa decompression and clot evacuation for fourth ventricle hemorrhage after aneurysmal rupture: case report. Neurosurgery 49:208-211, 2001

23. Lapointe M, Haines S: Fibrinolytic therapy for intraventricular hemorrhage in adults. Cochrane Database Syst Rev 3: CD003692, 2002

24. Lodhia KR, Shakui P, Keep RF: Hydrocephalus in a rat model of intraventricular hemorrhage. Acta Neurochir Suppl 96: 207-211, 2006

25. Longatti P, Fiorindi A, Martinuzzi A: Neuroendoscopic aspiration of hematocephalus totalis: technical note. Neurosurgery 57 (4 Suppl):E409, 2005

26. Longatti PL, Martinuzzi A, Fiorindi A, Maistrello L, Carteri A: Neuroendoscopic management of intraventricular hemorrhage. Stroke 35: $35-\mathrm{e} 38,2004$

27. Martí-Fàbregas J, Piles S, Guardia E, Martí-Vilalta JL: Spontaneous primary intraventricular hemorrhage: clinical data, etiology and outcome. J Neurol 246:287-291, 1999

28. Mayer SA, Kessler DB, Van Hurtum RL, Thomas CE, Fink ME, Brannigan T: Effect of intraventricular blood on global cortical perfusion in acute intracerebral hemorrhage: a singlephoton emission computed tomographic study. Ann Neurol 38:288, 1995 (Abstract)

29. Mayfrank L, Kissler J, Raoofi R, Delsing P, Weis J, Küker W, et al: Ventricular dilatation in experimental intraventricular hemorrhage in pigs. Characterization of cerebrospinal fluid dynamics and the effects of fibrinolytic treatment. Stroke 28:141-148, 1997

30. Morgan T, Awad I, Keyl P, Lane K, Hanley D: Preliminary report of the clot lysis evaluating accelerated resolution of intraventricular hemorrhage (CLEAR-IVH) clinical trial. Acta Neurochir Suppl 105:217-220, 2008

31. Naff NJ, Carhuapoma JR, Williams MA, Bhardwaj A, Ulatowski JA, Bederson J, et al: Treatment of intraventricular hemorrhage with urokinase: effects on 30-day survival. Stroke 31:841-847, 2000

32. Naff NJ, Williams MA, Rigamonti D, Keyl PM, Hanley DF: Blood clot resolution in human cerebrospinal fluid: evidence of first-order kinetics. Neurosurgery 49:614-621, 2001

33. Nakagawa T, Suga S, Mayanagi K, Akaji K, Inamasu J, Kawase $\mathrm{T}$ : Predicting the overall management outcome in patients with a subarachnoid hemorrhage accompanied by a massive intracerebral or full-packed intraventricular hemorrhage: a 15-year retrospective study. Surg Neurol 63:329-335, 2005

34. Nakano T, Ohkuma H, Ebina K, Suzuki S: Neuroendoscopic surgery for intracerebral haemorrhage-comparison with traditional therapies. Minim Invasive Neurosurg 46:278-283, 2003

35. Nieuwkamp DJ, de Gans K, Rinkel GJ, Algra A: Treatment and outcome of severe intraventricular extension in patients with subarachnoid or intracerebral hemorrhage: a systematic review of the literature. J Neurol 247:117-121, 2000
36. Nishikawa T, Takehira N, Matsumoto A, Kanemoto M, Kang Y, Waga S: Delayed endoscopic intraventricular hemorrhage (IVH) removal and endoscopic third ventriculostomy may not prevent consecutive communicating hydrocephalus if IVH removal was insufficient. Minim Invasive Neurosurg 50: 209-211, 2007

37. Nishikawa T, Ueba T, Kajiwara M, Iwata R, Yamashita K: Combined treatment of ruptured aneurysm accompanied by intraventricular hemorrhage; neuroendoscopy and coiling: case report. Minim Invasive Neurosurg 51:354-357, 2008

38. Oka K, Go Y, Yamamoto M, Kumate S, Tomonaga M: Experience with an ultrasonic aspirator in neuroendoscopy. Minim Invasive Neurosurg 42:32-34, 1999

39. Rainov NG, Burkert WL: Urokinase infusion for severe intraventricular haemorrhage. Acta Neurochir (Wien) 134:55-59, 1995

40. Rosen DS, Macdonald RL, Huo D, Goldenberg FD, Novakovic RL, Frank JI, et al: Intraventricular hemorrhage from ruptured aneurysm: clinical characteristics, complications, and outcomes in a large, prospective, multicenter study population. $\mathbf{J}$ Neurosurg 107:261-265, 2007

41. Rosenberg GA, Saland L, Kyner WT: Pathophysiology of periventricular tissue changes with raised CSF pressure in cats. J Neurosurg 59:606-611, 1983

42. Shapiro SA, Campbell RL, Scully T: Hemorrhagic dilation of the fourth ventricle: an ominous predictor. J Neurosurg 80:805-809, 1994

43. Shen PH, Matsuoka Y, Kawajiri K, Kanai M, Hoda K, Yamamoto S, et al: Treatment of intraventricular hemorrhage using urokinase. Neurol Med Chir (Tokyo) 30:329-333, 1990

44. Song Z, Yang QD, Zi XH, Fan X: Modified Graeb criteria for predicting the post-hemorrhagic hydrocephalus in intraventricular hemorrhage. Chin Med Sci J 19:138-141, 2004

45. Teasdale G, Jennett B: Assessment of coma and impaired consciousness. A practical scale. Lancet 2:81-84, 1974

46. Todo T, Usui M, Takakura K: Treatment of severe intraventricular hemorrhage by intraventricular infusion of urokinase. J Neurosurg 74:81-86, 1991

47. Tuhrim S, Horowitz DR, Sacher M, Godbold JH: Volume of ventricular blood is an important determinant of outcome in supratentorial intracerebral hemorrhage. Crit Care Med 27: 617-621, 1999

48. Tush GM, Tesoro EP, Prabhu S, Brown K, Bullock MR: Intraventricular urokinase for the treatment of intraventricular hemorrhage. Crit Care Med 27 (Suppl 1):79A, 1999 (Abstract)

49. Wasserman JK, Schlichter LC: White matter injury in young and aged rats after intracerebral hemorrhage. Exp Neurol 214:266-275, 2008

50. Young WB, Lee KP, Pessin MS, Kwan ES, Rand WM, Caplan LR: Prognostic significance of ventricular blood in supratentorial hemorrhage: a volumetric study. Neurology 40:616-619, 1990

51. Zhang Z, Li X, Liu Y, Shao Y, Xu S, Yang Y: Application of neuroendoscopy in the treatment of intraventricular hemorrhage. Cerebrovasc Dis 24:91-96, 2007

Manuscript submitted December 6, 2011.

Accepted January 16, 2012.

Please include this information when citing this paper: DOI: 10.3171/2012.1.FOCUS11349.

Address correspondence to: Luca Basaldella, M.D., Department of Neurosurgery, Treviso Regional Hospital, University of Padova, Piazzale Ospedale Civile 1, 31100 Treviso, Italy. email: lbasaldella@ulss.tv. 